score of periods have been determined from 25.5 to 27.5 days, depending somewhat upon the data employed and upon the method of its manipulation. It is very certain that, if any one will take the 'horizontal force,' for example, and arrange the observations in intervals of 26 days (the best thus far found) he will quickly find that, judging from the disturbed days, there is absolutely no fixed interval. These disturbed days would scem the very best material for such studies, as they are very definite. These days will occur for three or four rotations most beautifully, but after that the disturbance disappears and no more will appear along that line for a score of rotations.

In the same way one will very quickly find, in using the data and leaving out the disturbed days, that there is absolutely no recurring period of 26 days or any fraction of that interval. Sometimes by grouping ton rotations one will find a fairly good fluctuation, but the very next group of ten rotations will make 'hodge podge' of the previous group. This would seem an extremely important point to settle, as months have been devoted to fruitless efforts in trying to determine such a period.

3. The fluctuations under (c) above are simultaneous over the whole earth, as has been shown by the records at Batavia, India, Los Angeles, Cal.; St. Petersburg and Tiflis, in Russia; Vienna, Austria; Washington, D. C., and Zikawei, China. One is struck at once by the wonderful regularity of these fluctuations over the whole Northern Hemisphere. Making allowance for the difference of time and for disturbed days, the fluctuations are found to be exactly the same at each station, and the record at a single station will answer perfectly for comparison with any supposed related meteorologic phenomenon.

4. After thirteen years of study and careful discussion I am satisfied that the pressure of the air, or perhaps the fluctuations of the dew point, are by far the best to use for determining a possible connection with magnetism. I am also perfectly satisfied that, except in the cases specified above, there is no direct relation between magnetic and meteorologic phenomena, and this is also the outcome of the exhaustive studies in England and on the continent. I am also satisfied that there is an indirect relation, but the phenomena are so extremely complex that it has proven impossible to determine it up to the present.

5. In all studies of this character, and in all attempts at determining coincidences between such phenomena, one will always find a most valuable check by cutting up the long list of rotations into groups of 7 , 10 or 14 rotations in each. If these separate groups do not show a thread running through them, or fluctuations common to all and continually recurring, he may be satisfied that there is nothing in it. There is a peculiar and well-nigh unaccountable fascination in arranging and summing groups of figures in the hope that something may come, but continuous effort will show that there is something back of it all which is not understood, and no headway can be made by direct comparisons.

H. A. Hazen.

February 1, 1895.

\section{SIMILAR INIVENTIONS IN AREAS WIDE APART.}

As a contribution to the much disputed question of the occurrence of similar inventions in areas wide apart, I desire to call the attention of readers to the device for weaving of which I have found abundant examples in the Pueblo country, in the New England States, and in Finland.

The apparatus consists essentially of a small rectangular frame-work in which are placed a series of perpendicular slats perforated in the middle. It has the appearance 
of a grating of small bars about one-sixteenth of an inch apart, and each bar is pierced in the middle. In fact, all of these are the harness of a small loom used in weaving tape, braid, garters, belts and the like.

- Among the old-time families of New England, this apparatus is set up by taking a ball of twine or thread which is to constitute the warp, and walking around a number of chairs placed at a distance from one another as many times as there are to be threads in the warp. This coil is then cut apart, one end tied together in a knot, and the separate threads of the other end passed through the holes of the slats and between them. This apparatus is worked by lifting and depressing this frame as the weft shuttle is passed backward and forward by the hand. At each turn the weft is beaten home by the harness, the lower end of which is held between the knees, by the shuttle, or by the hand.

In a Zuñi example in the Museum set up by Mr. Cushing, the weaver sits upon the ground, having the far end of the warp fastened to some part of the building, and the proximal end attached to a stick forming part of a belt. The very same process employed by the New England woman is also in vogue among the Pueblos. By lifting and depressing the frame which is simply a couple of parallel sticks to which split reeds are tied, having holes burnt through the center, the weaver is able to pass the shuttle stick backward and forward.

When the Pueblo woman wishes to make short garters she uises the soles of her feet as a resting place for the little bar to which the far end of the weft is attached. Her shuttle is a stick on which the weft yarn is wound.

The Finnish harness is carved from a single block of wood, the upper and lower borders being somewhat cylindrical and the upright bars carved like little slats from the solid piece. These are perforated exactly after the manner of the New England examples.

I learn by inquiring at the Patent Office in Washington, that in Belgium a patent has recently been issued for an improvement on this style of weaving apparatus.

$I$ leave the question open as to the amount of contact between the Fins, the New England housewife and the Pueblo woman. It is easy enough to account for the dispersion of this apparatus among the white people of Europe, and thence among the Fins and the New England farmers. The only question for us to inquire into now, is, where did the Pueblo woman learn to weave after this fashion?

Dr. Matthews tells me that the Navajo do not use this frame, but make their belts by means of a harness similar to that which they employ in making their blankets. It is also a question where and how the Navajos learned to set up a loom so much like those found among the primitive European weavers. It is a fact that the Aino employ precisely the same apparatus as do the Navajo.

O. T. Mason.

WASHINGTON.

THE SOCIAL SEN'SE.

Alu persons thrown intimately with children from about four years of age and later may serve psychologists by making detailed observations of what may be called 'chumming' on the part of children and youth. By 'chumming' is meant all instances of unusually close companionship voluntarily made, 'platonic affection,' personal influence one over another when this influence is limited more or less to one person, and when the relationship is stronger than ordinary and is shown in any unusual or remarkable ways, such as bearing punishment for or with the other, moping or becoming very unsocial when separated. Cases of 